ESAIM: M2AN 49 (2015) 1-17

DOI: $10.1051 / \mathrm{m} 2 \mathrm{an} / 2014020$
ESAIM: Mathematical Modelling and Numerical Analysis

www.esaim-m2an.org

\title{
DIFFUSION LIMIT OF FOKKER-PLANCK EQUATION WITH HEAVY TAIL EQUILIBRIA *
}

\author{
Elissar NAsReddine ${ }^{1}$ And Marjolaine Puel ${ }^{2}$
}

\begin{abstract}
This paper is devoted to the diffusion limit of the Fokker-Planck equation of plasma physics, in which the equilibrium function decays towards zero at infinity like a negative power function. We prove that for an appropriate time scale, in a suitable weighted Sobolev space, the small mean free path limit gives rise to a diffusion equation.
\end{abstract}

Mathematics Subject Classification. 60J60, 60J70, 35Q84.

Received May 17, 2013. Revised February 17, 2014.

Published online January 12, 2015.

\section{INTRODUCTION}

We consider a collisonal kinetic equation given by

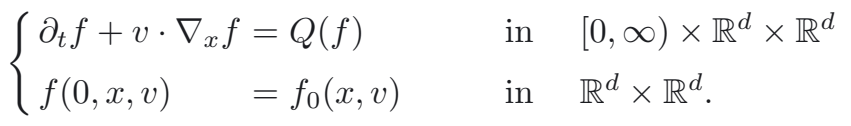

Such a problem naturally arises when modeling the behaviour of a cloud of particles. The unknown $f(t, x, v) \geq 0$ can be interpreted as the density of particles occupying at time $t \geq 0$, the position $x \in \mathbb{R}^{d}$ with a physical state described by the variable $v \in \mathbb{R}^{d}$. This variable $v$ represents the velocity of the particles.

We focus in this paper on the Fokker-Planck equation when the collisional operator $Q$ has a diffusive form:

$$
Q(f):=\nabla_{v} \cdot\left(\frac{1}{\omega} \nabla_{v}(f \omega)\right)
$$

and where the equilibria are characterised by the choice of $\omega$ (Indeed, this operator has one dimensional kernel spanned by the function $F(v)=\frac{K}{\omega}$ where $K$ is a normalizing constant). In this equation, the scattering in velocity is modeled by a diffusion phenomena coming from a deterministic formulation of a Brownian motion.

\footnotetext{
Keywords and phrases. Fokker Plank, diffusion limit, heavy tail, Cauchy distribution.

* This work is dedicated to the memory of Naoufel Ben Abdallah, who proposed this subject to E.N. during her Master degree.

1 Institut de Mathématiques de Toulouse, Université de Toulouse, 31062 Toulouse cedex 9, France.

elissar.nasreddine@math.univ-toulouse.fr

2 Laboratoire Dieudonné, Université de Nice Sophia Antipolis, Parc Valrose, 06108 Nice cedex 2, France.

Marjolaine.Puel@unice.fr
} 
Another classical example of collision operator is given by the linear (semiconductor) Boltzmann equation where $Q$ is given by an integral operator

$$
Q(f)=\int_{\mathbb{R}^{d}} \sigma\left(v, v^{\prime}\right)\left[f\left(v^{\prime}\right) F(v)-f(v) F\left(v^{\prime}\right)\right] \mathrm{d} v^{\prime}, \quad \text { with } \sigma\left(v, v^{\prime}\right)=\sigma\left(v^{\prime}, v\right),
$$

where $F(v)$ denotes the equilibrium, $\sigma$ is the scattering function and $\sigma\left(v, v^{\prime}\right) F(v)$ models the probability of a particle to pass from a velocity $v^{\prime}$ before the collision to velocity $v$ after the collision.

For both problems, when the scattering phenomena is much stronger than the advection phenomena, it is possible to approximate the solution of (1.1) by a density depending only on the time and space variable multipied by a velocity profile given by the thermodynamical equilibrium. For example, when the equilibria are given by Maxwellian (or Gaussian) distribution functions, the density is proved to satisfy a diffusion equation. This process is known as the diffusion approximation and has been investigated for a long time, starting with Bensoussan et al. [4] and Larsen and Keller [12] and it has been the topic of many papers since then (see in particular Bardos et al. [1] and Degond et al. [10] and references therein or Lions and Toscani [18] who obtain non linear diffusion equation). In the case of the linear Boltzmann problem, (see Ben Abdallah et al. [2,3], Mellet et al. [16] Mellet [17]) similar asymptotic analysis are performed when the equilibrium function is not a Maxwellian distribution, but rather a heavy tail function. Depending on the power of the tail, the diffusion coefficient involved in the equation satisfied by the density is well defined or not. When it is not well defined, we talk about anomalous diffusion and after an ad hoc rescaling, the density satisfies a non local equation such as a fractional diffusion equation.

In the present paper, we also consider heavy tail equilibria $F(v)=\frac{K}{\omega}$ corresponding to $\omega=\left(1+\|v\|^{2}\right)^{\frac{\beta}{2}}$ for $\beta>d$, and we obtain a classical diffusion equation in the case where the diffusion coefficient is well defined. In the Fokker Planck context, the heavy tail functions also called Cauchy distribution family are considered as equilibria in the case where Fokker Plank is slightly modified by taking fractional Fokker Planck ([6]) but also as target for the usual Fokker Planck equation. For instance, those profile appear as equilibria for the Fokker Planck equation satisfied by Wigner function in the anomalous quantum transport in optical lattice (for physical references, see $[11,14,15])$. The main difference with Boltzmann is the functional setting, and this difficulty is the reason why the case of anomalous diffusion is still not clear.

Mathematically speaking, we introduce a small parameter $\varepsilon \ll 1$ which describes the mean free path of the particles, then we consider the following rescaling

$$
x^{\prime}=\varepsilon x \text { and } t^{\prime}=\theta(\varepsilon) t, \text { with } \theta(\varepsilon) \rightarrow 0 .
$$

Typically, it means that we assume that the mean free path is very small and the time scale is very large. Then, we rescale the distribution function

$$
f^{\varepsilon}\left(t^{\prime}, x^{\prime}, v\right)=f(t, x, v) .
$$

The function $f^{\varepsilon}$ is now solution of (we skipped the primes)

$$
\begin{aligned}
\theta(\varepsilon) \partial_{t} f^{\varepsilon}+\varepsilon v \cdot \nabla_{x} f^{\varepsilon} & =Q\left(f^{\varepsilon}\right), \\
f^{\varepsilon}(0, x, v) & =f_{0}(x, v) .
\end{aligned}
$$

The goal is then to study the behaviour of the solution as $\varepsilon \rightarrow 0$.

The usual diffusion limit correspond to $\theta(\varepsilon)=\varepsilon^{2}$. It may be formally studied using the so-called Hilbert expansion method (see $[8,9]$ ), which is based on a formal expansion of the solution in the form

$$
f^{\varepsilon}=f^{0}+\varepsilon f^{1}+\varepsilon^{2} f^{2} .
$$

Inserting this expansion in (1.4) and identifying the term of same order in $\varepsilon$ yields to a diffusion equation verified by $\rho(t, x)=\int f^{0} \mathrm{~d} v$, see Section 3 . 
To make the formal proof rigorous, we thus propose a different method, the moment method classically used to obtain weak convergence in the study of limits of kinetic equations under weaker assumptions on the initial data. This method relies on the introduction of an appropriate auxiliary problem and a weak formulation of (1.4) and (1.2). More precisely, the corner stone of the proof is to multiply equation (1.4) by a test function that will give us an equation for the density. We will see that an ad hoc choice is given by $\phi(t, x) F(v)+\varepsilon \psi(t, x, v)$, where the correction $\psi$ is the solution of the auxiliary equation

$$
Q(\psi)=-F(v) v \cdot \nabla_{x} \phi
$$

where $\phi$ is a smooth test function. Note the presence of $F(v)$ due to the fact that we use weighted Sobolev spaces with a duality product involving the factor $\frac{1}{F(v)}$. Thanks to the moment method, we prove that for $\theta(\varepsilon)=\varepsilon^{2}$, the function $f^{\varepsilon}(t, x, v)$ converges weakly, when $\varepsilon$ goes to zero, to a function of the form $\rho F(v)$ where the density $\rho(t, x)$ solves the diffusion equation:

$$
\partial_{t} \rho-\nabla_{x} \cdot\left(D \nabla_{x} \rho\right)=0 .
$$

This convergence is proved under some assumptions on $F$ that guarantee that the diffusion tensor $D$, which depends on $F$, is finite, namely $\beta>d+4$, see Theorem 2.2 below.

The case $\beta=d+4$, is critical in the sense that the diffusion coefficient obtained in this paper is no more defined. Even though, we may obtain an asymptotic by changing the scaling as in the Boltzmann case. Indeed, we proved in collaboration with Cattiaux [5] that with the time scale $\theta(\varepsilon)=\varepsilon^{2}|\ln \varepsilon|$ will still obtain a diffusion equation. We can't say what happens for $\beta<d+4$, the asymptotic may lead to a different phenomena or not.

From now on, we assume that

$$
Q(f):=\nabla_{v} \cdot\left(\frac{1}{\omega} \nabla_{v}(f \omega)\right)
$$

where $\omega=\left(1+\|v\|^{2}\right)^{\frac{\beta}{2}}$ and $\beta>d$ and we denote $K>0$ the constant renormalizing the equilibrium; i.e. satisfying $\int_{\mathbb{R}^{d}} \frac{K}{\omega} \mathrm{d} v=1$.

\section{MAIN RESULTS}

We start with an existence result. For that purpose, let us define the functional spaces $Y_{\omega}^{p}\left(\mathbb{R}^{2 d}\right)=$ $L^{p}\left(\mathbb{R}^{d}, H_{p}\left(\mathbb{R}^{d}\right)\right)$, where

$$
H_{p}\left(\mathbb{R}^{d}\right)=\left\{f: \mathbb{R}^{d} \rightarrow \mathbb{R}, \int_{\mathbb{R}^{d}}|f|^{p} \omega^{p-1} \mathrm{~d} v<\infty\right\}
$$

where $\omega=\left(1+\|v\|^{2}\right)^{\frac{\beta}{2}}$ and

$$
V=\left\{f: \mathbb{R}^{d} \rightarrow \mathbb{R}, \int_{\mathbb{R}^{d}}|f|^{2} \omega \mathrm{d} v<\infty \text { and } \int_{\mathbb{R}^{d}} \frac{\left|\nabla_{v}(f \omega)\right|^{2}}{\omega} \mathrm{d} v<\infty\right\},
$$

$V^{\prime}$ being its dual. Thanks to Lions's theorem [13] we obtain the following theorem

Theorem 2.1. Assume that $f_{0} \in Y_{\omega}^{2}\left(\mathbb{R}^{d}\right)$, the system (1.1) has a unique solution $f$ in the class of functions $Y$ defined by:

$$
Y=\left\{f \in L^{2}\left([0, T] \times \mathbb{R}^{d}, V\right), \quad \partial_{t} f+v \cdot \nabla_{x} f \in L^{2}\left([0, T] \times \mathbb{R}^{d}, V^{\prime}\right)\right\} .
$$

As we said, passing from the microscopic to the macroscopic scales relies on a rescaling of (1.1) involving the small parameter $\varepsilon \ll 1$. The study of the behaviour of the solution as $\varepsilon \rightarrow 0$ is the object of our main result:

Theorem 2.2. Assume that $f_{0}$ is a nonnegative function in $Y_{\omega}^{2} \cap Y_{\omega}^{p}$ with $p>2$ and $\beta>d+4$. Let $f^{\varepsilon}$ be the solution of (1.4) in $Y$ with initial data $f_{0}$, when $\theta(\varepsilon)=\varepsilon^{2}$. 
Then, $f^{\varepsilon}$ converges weakly star in $L^{\infty}\left([0, T], Y_{\omega}^{p}\left(\mathbb{R}^{2 d}\right)\right)$ towards $\rho(t, x) \frac{K}{\omega}$ where $\rho(t, x)$ is the unique solution of the system

$$
\begin{gathered}
\partial_{t} \rho+\nabla_{x} \cdot j=0 \\
j=-D \nabla_{x} \rho,
\end{gathered}
$$

where the initial datum is given by $\rho_{0}(x)=\int_{\mathbb{R}^{d}} f_{0} \mathrm{~d} v$, and the diffusion tensor $D$ is given by

$$
D=\int_{\mathbb{R}^{d}} v \otimes \chi \mathrm{d} v
$$

where $\chi$ is the unique solution of the cell equation $Q(\chi)=\frac{-K v}{\omega}$ with $\int_{\mathbb{R}^{d}} \chi \mathrm{d} v=0$.

\section{Remark 2.3.}

1. We can remark that the diffusion tensor $D$ in (2.5) has the same form of Boltzmann coefficient,

$$
D=\int_{\mathbb{R}^{d}} v \otimes \chi \mathrm{d} v, \quad Q(\chi)=\frac{-K v}{\omega},
$$

but here, $\chi$ can be substituted by its explicit form (5.2).

2. The most difficult point in this problem is the introduction of weighted functional spaces in the proof of the convergence result. Indeed, it involves duality products that degenerate in the critical case where $\beta=d+4$ which leads to an additional difficulty in the study of the anomalous diffusion.

The paper is organized as follows: in Section 3, we prove existence and uniqueness for equations (1.1). Then, in Section 4, we derive formally the diffusion equation satisfied by the limiting density using Hilbert expansion. Section 5 is devoted to the study of the auxiliary problem leading to $\chi$ and of the diffusion coefficient $D$. The final step is the obtention of the convergence via moment method and is performed in Section 6 .

\section{Functional Setting And EXistence Result}

\subsection{Properties of the collision operator and adapted functional setting}

The following proposition gives some properties of the collision operator $Q$.

Proposition 3.1. Let $f$ and $g$ be smooth functions in $V$ defined in (2.2). The following assertions hold true:

1. The operator $Q$ is conservative, thus equation (1.4) preserves the total mass of the distribution

$$
\int_{\mathbb{R}^{d}} Q(f) \mathrm{d} v=0, \text { for all } f \in V .
$$

2. The operator $Q$ is self-adjoint with respect to the measure $\omega \mathrm{d} v$ :

$$
\int_{\mathbb{R}^{d}} Q(f) g \omega \mathrm{d} v=-\int_{\mathbb{R}^{d}} \frac{\nabla_{v}(f \omega) \cdot \nabla_{v}(g \omega)}{\omega} \mathrm{d} v=\int_{\mathbb{R}^{d}} f Q(g) \omega \mathrm{d} v
$$

3. The operator $Q$ is dissipative:

$$
\int_{\mathbb{R}^{d}} Q(f) f \omega \mathrm{d} v=-\int_{\mathbb{R}^{d}} \frac{\left|\nabla_{v}(f \omega)\right|^{2}}{\omega} \mathrm{d} v \leq 0
$$

4. The kernel of $Q$ is one-dimensional and spanned by $F(v)=\frac{K}{\omega}$ ( $K$ being a normalizing constant).

5. The operator $Q$ is continuous from $V \longrightarrow V^{\prime}$. 
Proof. In this proof we show 4 and 5.

1. It is clear that $\frac{\mathbb{R}}{\omega} \subset \operatorname{Ker}(Q)$.

For $f \in V$ such that $Q(f)=0$ then for any $\varphi \in D\left(\mathbb{R}^{d}\right)$ we have:

$$
\int_{\mathbb{R}^{d}} \nabla_{v} \cdot\left(\frac{1}{\omega} \nabla_{v}(f \omega)\right) \varphi \mathrm{d} v=0
$$

therefore

$$
\int_{\mathbb{R}^{d}} \frac{\nabla_{v}(f \omega) \cdot \nabla_{v}(\varphi \omega)}{\omega} \mathrm{d} v=0 .
$$

Since $D\left(\mathbb{R}^{d}\right)$ is dense in $V$, then $\int_{\mathbb{R}^{d}} \frac{\left|\nabla_{v}(f \omega)\right|^{2}}{\omega} \mathrm{d} v=0$ and there exists a constant $\rho \in \mathbb{R}$ such that $f=\frac{\rho}{\omega}$ and we obtain $\operatorname{Ker} Q \subset \frac{\mathbb{R}}{\omega}$.

2. Using Cauchy-Schwarz inequality we obtain that for a test function $\phi \in D\left(\mathbb{R}^{d}\right)$ and $f \in V$,

$$
\begin{aligned}
\left|\int_{\mathbb{R}^{d}} Q(f) \phi \mathrm{d} v\right| & \leq \int_{\mathbb{R}^{d}}\left|\frac{\nabla_{v}(f \omega) \cdot \nabla_{v}(\phi \omega)}{\omega}\right| \mathrm{d} v \\
& \leq\left.\left\|\frac{\nabla_{v}(f \omega)}{\omega^{\frac{1}{2}}}\right\|\right|_{2}\left\|\frac{\nabla_{v}(\phi \omega)}{\omega^{\frac{1}{2}}}\right\|_{2} \\
& \leq\|f\|_{V}\|\phi\|_{V} .
\end{aligned}
$$

Therefore

$$
\|Q(f)\|_{V^{\prime}} \leq\|f\|_{V}
$$

hence the continuity holds.

Proposition 3.1 shows that the natural $L^{2}$ norm associated with this operator has a weight $\omega$ and that the $H^{1}$ semi norm is given by the right-hand side of (3.2). This motivates the introduction of the following functional spaces, endowed with their naturally associated norms:

$$
\begin{gathered}
H=\left\{f: \mathbb{R}^{d} \rightarrow \mathbb{R} ; \int_{\mathbb{R}^{d}}|f|^{2} \omega \mathrm{d} v<\infty\right\}, \quad \text { and }\langle\cdot, \cdot\rangle_{H} \text { the associated scalar product } \\
V=\left\{f: \mathbb{R}^{d} \rightarrow \mathbb{R} ; \int_{\mathbb{R}^{d}}|f|^{2} \omega \mathrm{d} v<\infty \text { and } \int_{\mathbb{R}^{d}} \frac{\left|\nabla_{v}(f \omega)\right|^{2}}{\omega} \mathrm{d} v<\infty\right\}, \\
H_{p}=\left\{f: \mathbb{R}^{d} \rightarrow \mathbb{R} ; \int_{\mathbb{R}^{d}}|f|^{p} \omega^{p-1} \mathrm{~d} v<\infty\right\}, \\
V_{p}=\left\{f \in H_{p} ; \int_{\mathbb{R}^{d}}\left|\nabla_{v}(f \omega)\right|^{p} \omega^{-p+1} \mathrm{~d} v<\infty\right\}, \\
X=L^{2}\left([0, T] \times \mathbb{R}_{x}^{d}, V\right) ; Y_{\omega}^{p}\left(\mathbb{R}^{2 d}\right)=L^{p}\left(\mathbb{R}_{x}^{d}, H_{p}\right) ; \quad X_{p}=L^{2}\left([0, T] \times \mathbb{R}_{x}^{d}, V_{p}\right) .
\end{gathered}
$$

Now, we are in a position to define the dual space of $Y_{\omega}^{p}\left(\mathbb{R}^{2 d}\right)$. 
Lemma 3.2. For $p \in\left[1, \infty\left[\right.\right.$, define $p^{\prime}$ by $\frac{1}{p}+\frac{1}{p^{\prime}}=1$. Then $Y_{\omega}^{p^{\prime}}\left(\mathbb{R}^{2 d}\right)$ is the dual space of $Y_{\omega}^{p}\left(\mathbb{R}^{2 d}\right)$.

Proof. Let us consider that $f \in Y_{\omega}^{p}\left(\mathbb{R}^{2 d}\right)$ and $g \in Y_{\omega}^{p^{\prime}}\left(\mathbb{R}^{2 d}\right)$, then Hölder inequality gives that:

$$
\begin{aligned}
\left|\int_{\mathbb{R}^{2 d}} f g \omega \mathrm{d} v \mathrm{~d} x\right| & \leq \int_{\mathbb{R}^{2 d}}\left(|f| \omega^{\frac{p-1}{p}}\right)\left(|g| \omega^{\frac{-(p-1)}{p}} \omega\right) \mathrm{d} v \mathrm{~d} x \\
& \leq \int_{\mathbb{R}^{2 d}}\left(|f| \omega^{\frac{p-1}{p}}\right)\left(|g| \omega^{\frac{p^{\prime}-1}{p^{\prime}}}\right) \mathrm{d} v \mathrm{~d} x \\
& \leq\left(\int_{\mathbb{R}^{2 d}}|f|^{p} \omega^{p-1} \mathrm{~d} v \mathrm{~d} x\right)^{\frac{1}{p}}\left(\int_{\mathbb{R}^{2 d}}|g|^{p^{\prime}} \omega^{p^{\prime}-1} \mathrm{~d} v \mathrm{~d} x\right)^{\frac{1}{p^{\prime}}} \\
& =\|f\|_{Y_{\omega}^{p}}\|g\|_{Y_{\omega}^{p^{\prime}}}
\end{aligned}
$$

which gives the result.

\subsection{Existence and uniqueness}

In this section we prove the theorem establishing the existence and uniqueness of a solution to (1.1). The proof relies to Lions theorem see $[7,13]$.

Proof of Theorem 2.1. For any $\lambda>0$, the change of unknown:

$$
f_{\lambda}(x, v, t)=\mathrm{e}^{-\lambda t} f(t, x, v)
$$

leads to the equation:

$$
\left\{\begin{aligned}
\partial_{t} f_{\lambda}+v \cdot \nabla_{x} f_{\lambda}+\lambda f_{\lambda} & =\nabla_{v} \cdot\left(\frac{1}{\omega} \nabla_{v}\left(f_{\lambda} \omega\right)\right) \\
f_{\lambda}(0, x, v) & =f_{0}(x, v) .
\end{aligned}\right.
$$

We will prove existence and uniqueness for $f_{\lambda}$.

Let

$$
X=L^{2}\left([0, T] \times \mathbb{R}^{d}, V\right), \text { and }\|\varphi\|_{X}^{2}=\int_{0}^{T} \int_{\mathbb{R}^{d}} \int_{\mathbb{R}^{d}}|\varphi(t, x, v)|^{2} \omega \mathrm{d} v \mathrm{~d} x \mathrm{~d} t
$$

and $S$ be the space $] 0, T\left[\times \mathbb{R}^{d} \times \mathbb{R}^{d}\right.$ ) of infinitely differentiable functions, with compact support in $] 0, T\left[\times \mathbb{R}^{d} \times \mathbb{R}^{d}\right.$, provided with Hilbertian norm:

$$
\|\varphi\|_{S}^{2}=\|\varphi\|_{X}^{2}+\frac{1}{2} \int_{\mathbb{R}^{d}} \int_{\mathbb{R}^{d}} \omega|\varphi(0, x, v)|^{2} \mathrm{~d} v \mathrm{~d} x, \quad \forall \varphi \in S .
$$

The bilinear form $E$, and the linear form $L$, are defined by:

$$
\begin{gathered}
E\left(f_{\lambda}, \varphi\right)=\int_{0}^{T} \int_{\mathbb{R}^{d}} \int_{\mathbb{R}^{d}}\left[f_{\lambda} \omega\left(-\frac{\partial \varphi}{\partial t}-v \cdot \nabla_{x} \varphi+\lambda \varphi\right)+\frac{1}{\omega} \nabla_{v}(\varphi \omega) \cdot \nabla\left(f_{\lambda} \omega\right)\right] \mathrm{d} v \mathrm{~d} x \mathrm{~d} t \\
L(\varphi)=\int_{\mathbb{R}^{d}} \int_{\mathbb{R}^{d}} f_{0}(x, v) \varphi(0, x, v) \omega \mathrm{d} v \mathrm{~d} x .
\end{gathered}
$$


The bilinear form $E$ is coercive since

$$
\begin{aligned}
E(\varphi, \varphi)= & \int_{0}^{T} \int_{\mathbb{R}^{d}} \int_{\mathbb{R}^{d}}\left[\varphi \omega\left(-\partial_{t} \varphi-v \cdot \nabla_{x} \varphi+\lambda \varphi\right)+\frac{1}{\omega} \nabla_{v}(\varphi \omega) \cdot \nabla(\varphi \omega)\right] \mathrm{d} v \mathrm{~d} x \mathrm{~d} t \\
= & \lambda \int_{0}^{T} \int_{\mathbb{R}^{d}} \int_{\mathbb{R}^{d}} \omega|\varphi|^{2} \mathrm{~d} v \mathrm{~d} x \mathrm{~d} t+\frac{1}{2} \int_{\mathbb{R}^{d}} \int_{\mathbb{R}^{d}} \omega|\varphi(0, x, v)|^{2} \mathrm{~d} v \mathrm{~d} x \\
& +\int_{0}^{T} \int_{\mathbb{R}^{d}} \int_{\mathbb{R}^{d}} \frac{\left|\nabla_{v}(\varphi \omega)\right|^{2}}{\omega} \mathrm{d} v \mathrm{~d} x \mathrm{~d} t \\
\geq & \min (\lambda, 1)\|\varphi\|_{X}^{2}+\frac{1}{2} \int_{\mathbb{R}^{d}} \int_{\mathbb{R}^{d}} \omega|\varphi(0, x, v)|^{2} \mathrm{~d} v \mathrm{~d} x \\
\geq & \min (\lambda, 1)\|\varphi\|_{S}^{2}
\end{aligned}
$$

Then Lions's theorem applies and the variational equation $E\left(f_{\lambda}, \varphi\right)=L(\varphi)$ admits a solution $f_{\lambda}$ in $X$. Then $f_{\lambda}$ satisfies (1.1) in the sense of distributions, and in particular, we deduce that:

$$
\frac{\partial f_{\lambda}}{\partial t}+v \cdot \nabla_{x} f_{\lambda}=\nabla_{v} \cdot\left(\frac{1}{\omega} \nabla_{v}\left(f_{\lambda} \omega\right)\right)-\lambda f_{\lambda} \in L^{2}\left([0, T] \times \mathbb{R}_{x}^{d}, V^{\prime}\right)
$$

so that $f_{\lambda}$ and then $f$ belong to $Y$.

In order to give a meaning to the initial condition, and to show uniqueness, we have to prove a trace theorem, and a Green formula for the functions of $Y$. Consequently, we admit the following lemma whose proof is a straightforward adaptation of [7] in the weighted Sobolev spaces. And for this reason, the proof is omitted.

Notations: In the following $\langle\cdot, \cdot\rangle_{X^{\prime} X}$ denotes the duality bracket between the space $X$ and its dual.

Lemma 3.3. (i) If $f \in Y, f$ admits (continuous) trace value $f(0, x, v)$ in $Y_{\omega}^{2}\left(\mathbb{R}^{2 d}\right)$.

(ii) For $f_{1}$ and $f_{2}$ in $Y$, we have:

$$
\left\langle\frac{\partial f_{1}}{\partial t}+v \cdot \nabla_{x} f_{1}, f_{2}\right\rangle_{X^{\prime} X}+\left\langle\frac{\partial f_{2}}{\partial t}+v \cdot \nabla_{x} f_{2}, f_{1}\right\rangle_{X^{\prime} X}=-\iint f_{1}(0, x, v) f_{2}(0, x, v) \omega \mathrm{d} v \mathrm{~d} x .
$$

So, using equation (3.8) and the Green formula (3.10), we deduce that the solution $f$ satisfies:

$$
\int_{\mathbb{R}^{d}} \int_{\mathbb{R}^{d}}\left[f(0, x, v)-f_{0}(x, v)\right] \varphi(0, x, v) \omega \mathrm{d} v \mathrm{~d} x=0, \quad \forall \varphi \in S .
$$

Consequently, the initial condition is satisfied in $Y_{\omega}^{2}\left(\mathbb{R}^{2 d}\right)$.

Now, for uniqueness, we suppose that $f_{1}$ and $f_{2}$ are two differents solutions of (3.8). Then we get that $f=f_{1}-f_{2}$ is a solution of (3.8) with $f_{0}=0$, which belongs to $Y$. Applying (3.10) we obtain:

$$
\begin{aligned}
0 & =\left\langle\frac{\partial f}{\partial t}+v \cdot \nabla_{x} f, f\right\rangle_{X^{\prime} X}-\left\langle\nabla_{v} \cdot\left(\frac{1}{\omega} \nabla_{v}(f \omega)\right), f\right\rangle_{X^{\prime} X}+\lambda\langle f, f\rangle_{X^{\prime} X} \\
& \geq \lambda \int_{0}^{T} \int_{\mathbb{R}^{2 d}}|f|^{2} \omega \mathrm{d} v \mathrm{~d} x \mathrm{~d} t .
\end{aligned}
$$

Which proves that $f$ is equal to zero almost everywhere.

\section{Formal ASYMptotics}

In this section we give a formal heuristic of Theorem 2.2. Recall that we investigate the asymptotic behaviour as $\varepsilon$ goes to zero of the solution of equation (1.4), when $\beta>d+4$ and $\theta(\varepsilon)=\varepsilon^{2}$.

The formal limit $\varepsilon \rightarrow 0$ can be seen following two points of view. 
We can perform the Hilbert expansion (see e.g. [8] for the general theory or [9] for an application in the context of Fokker-Planck equations)

$$
f^{\varepsilon}=f^{0}+\varepsilon f^{1}+\varepsilon^{2} f^{2}
$$

with $f^{k}$ being independent of $\varepsilon$. Inserting it into (1.4) and identifying terms having the same power of $\varepsilon$, we obtain the following set of equations, in which the $x$ variable is a parameter:

$$
\begin{gathered}
Q\left(f^{0}\right)=0, \\
Q\left(f^{1}\right)=v \cdot \nabla_{x} f^{0}, \\
Q\left(f^{2}\right)=\partial_{t} f^{0}+v \cdot \nabla_{x} f^{1} .
\end{gathered}
$$

Equation (4.1) means that $f^{0}$ lies in the Kernel of $Q$, and by Proposition 3.1

$$
f^{0}=\frac{\rho(t, x) K}{\left(1+\|v\|^{2}\right)^{\frac{\beta}{2}}},
$$

where $\rho(t, x)$ is a function still to be determined.

Since $f_{0}$ is even with respect to $v$, the Fredholm alternative says that equation (4.2) leads to

$$
f^{1}=-\chi \cdot \nabla_{x} \rho \quad \text { where } \quad \chi=Q^{-1}\left(\frac{-v K}{\left(1+\|v\|^{2}\right)^{\frac{\beta}{2}}}\right) .
$$

In order to determine $\rho$, we integrate (4.3) with respect to $v \in \mathbb{R}^{d}$ and use that $\int_{\mathbb{R}^{d}} Q(f) \mathrm{d} v=0$.

We get

$$
\partial_{t} \rho+\nabla_{x} \cdot\left(\int_{\mathbb{R}^{d}} v f_{1} \mathrm{~d} v\right)=0
$$

that is system (2.3), (2.4).

We can also define the density $\rho^{\varepsilon}(t, x)$ and the current $j^{\varepsilon}(t, x)$ by

$$
\rho^{\varepsilon}(t, x)=\int_{\mathbb{R}^{d}} f^{\varepsilon} \mathrm{d} v, \quad j^{\varepsilon}(t, x)=\frac{1}{\varepsilon} \int_{\mathbb{R}^{d}} v f^{\varepsilon} \mathrm{d} v .
$$

By integrating equation (1.4), we find

$$
\partial_{t} \rho^{\varepsilon}+\nabla_{x} \cdot j^{\varepsilon}=0
$$

Note that this equation is valid for all values of $\varepsilon$. Then we write the formal expansion $f^{\varepsilon}=f_{0}+\varepsilon f_{1}$.

Integrating it with respect to $v$, letting $\varepsilon \rightarrow 0$, we formally have $\rho^{\varepsilon} \rightarrow \rho$ since $\int_{\mathbb{R}^{d}} \frac{K}{\left(1+\|v\|^{2}\right)^{\frac{\beta}{2}}} \mathrm{~d} v=1$. Moreover, we have

$$
\begin{aligned}
j^{\varepsilon} & =\frac{1}{\varepsilon} \int_{\mathbb{R}^{d}} f^{0} v \mathrm{~d} v+\int_{\mathbb{R}^{d}} f^{1} v \mathrm{~d} v+O(\varepsilon) \\
& =0+\int_{\mathbb{R}^{d}} f^{1} v \mathrm{~d} v+O(\varepsilon),
\end{aligned}
$$

because $f^{0}$ is even in $v$. Therefore $j^{\varepsilon}$ has a limit when $\varepsilon \rightarrow 0$, and this limit is given by

$$
j(t, x)=\int_{\mathbb{R}^{d}} f^{1} v \mathrm{~d} v=\int_{\mathbb{R}^{d}}-v \chi \cdot \nabla_{x} \rho \mathrm{d} v .
$$

To make this proof rigorous, we need to justify all the formal convergence. That is what we are going to do using the moment method which consists in integrating the equation against suitable test functions. 


\section{Preliminary computations}

\subsection{Study of auxiliary equation}

We first compute $\chi$, the solution of the auxiliary problem satisfying

$$
Q(\chi)=-v F(v) .
$$

Then we give some properties of $\chi$.

Lemma 5.1. The unique solution of the cell equation

$$
Q(\chi)=\nabla_{v} \cdot\left(\frac{1}{\omega} \nabla_{v}(\chi \omega)\right)=-\frac{v K}{\omega} \text { with } \int_{\mathbb{R}^{d}} \chi \mathrm{d} v=0
$$

is given by:

$$
\chi=\frac{\|v\|^{2}+3}{3 \beta-2(d+2)} \frac{K v}{\omega} .
$$

Proof. First, since $F(v)=\frac{K}{\omega}$ is even with respect to $v$, the Fredholm alternative implies that there exists a solution to (5.1) which is unique as soon as we impose the constraint $\int_{\mathbb{R}^{d}} \chi \mathrm{d} v=0$. In order to compute the solution of (5.1), we use the following ansatz

$$
\chi=\frac{\varphi\left(\|v\|^{2}\right)}{\omega} v K
$$

where $\varphi$ is a function to determine.

First of all, we write

$$
\frac{\mathrm{d}}{\mathrm{d} v_{j}}\left(\varphi\left(\|v\|^{2} v_{i}\right)\right)=v_{i} \frac{\mathrm{d}}{\mathrm{d} v_{j}} \varphi\left(\|v\|^{2}\right)+\delta_{i j} \varphi\left(\|v\|^{2}\right)
$$

Then we get

$$
\begin{aligned}
\frac{\partial}{\partial v_{j}}\left(\frac{1}{\omega} \frac{\partial}{\partial v_{j}}\left(\varphi\left(\|v\|^{2} v_{i}\right)\right)\right)= & \frac{\partial}{\partial v_{j}}\left[\frac{v_{i}}{\omega} \frac{\partial}{\partial v_{j}} \varphi\left(\|v\|^{2}\right)+\frac{\delta_{i j}}{\omega} \varphi\left(\|v\|^{2}\right)\right] \\
= & \frac{\partial}{\partial v_{j}}\left(\frac{v_{i}}{\omega}\right) \frac{\partial}{\partial v_{j}} \varphi\left(\|v\|^{2}\right)+\frac{v_{i}}{\omega} \frac{\partial^{2}}{\partial^{2} v_{j}} \varphi\left(\|v\|^{2}\right) \\
& +\frac{\delta_{i j}}{\omega} \frac{\partial}{\partial v_{j}} \varphi\left(\|v\|^{2}\right)+\delta_{i j} \frac{\partial}{\partial v_{j}}\left(\frac{1}{\omega}\right) \varphi\left(\|v\|^{2}\right)
\end{aligned}
$$

Assume now that $\varphi\left(\|v\|^{2}\right)=a\|v\|^{2}+b$, we simplify the computation by writing

$$
\begin{aligned}
\frac{\partial}{\partial v_{j}}\left(\frac{1}{\omega} \frac{\partial}{\partial v_{j}}\left(\varphi\left(\|v\|^{2} v_{i}\right)\right)\right) & =\frac{\partial}{\partial v_{j}}\left(\frac{v_{i}}{\omega}\right)\left(2 a v_{j}\right)+\frac{v_{i}}{\omega} 2 d a+\frac{2 a v_{i}}{\omega}+\frac{\partial}{\partial v_{i}}\left(\frac{1}{\omega}\right)\left(a\|v\|^{2}+b\right) \\
& =\frac{4 a v_{i}+2 a \mathrm{~d} v_{i}}{\omega}+\frac{\partial}{\partial v_{i}}\left(\frac{1}{\omega}\right)\left(a\|v\|^{2}+b\right)+2 a v_{i} v_{j} \frac{\partial}{\partial v_{j}}\left(\frac{1}{\omega}\right)
\end{aligned}
$$

which leads to a rewriting of equation (5.1)

$$
\frac{v_{i}(4 a+2 d a)}{\left(1+\|v\|^{2}\right)^{\frac{\beta}{2}}}-\frac{2 \beta\|v\|^{2} v_{i} a}{\left(1+\|v\|^{2}\right)^{\frac{\beta}{2}+1}}-\frac{\beta v_{i}\left(a\|v\|^{2}+b\right)}{\left(1+\|v\|^{2}\right)^{\frac{\beta}{2}}}=\frac{-v_{i}}{\left(1+\|v\|^{2}\right)^{\frac{\beta}{2}}} .
$$


Then we get by identification

$$
a=\frac{-1}{-3 \beta+2(d+2)} \text { and } b=\frac{-3}{-3 \beta+2(d+2)} .
$$

We finally obtain

$$
\chi=\frac{\|v\|^{2}+3}{3 \beta-2(d+2)} \frac{K v}{\omega} .
$$

In the following lemma we study some properties of $\chi$.

Lemma 5.2. Recall that $H_{q}$ is defined by:

$$
H_{q}=\left\{f: \mathbb{R}^{d} \rightarrow \mathbb{R} ; \int_{\mathbb{R}^{d}}|f|^{q} \omega^{q-1} \mathrm{~d} v<\infty\right\}
$$

1. For $q<\frac{\beta-d}{3}$ we have $\chi \in H_{q}$.

2. For $q<\frac{\beta-d}{4}$ we have $v \cdot \chi \in H_{q}$.

Proof.

1. Let us rewrite

$$
\int_{\mathbb{R}^{d}}|\chi|^{q} \omega^{q-1} \mathrm{~d} v \leq \int_{\mathbb{R}^{d}}\left(\frac{\|v\|^{2}+3}{3 \beta-2(d+2)} \frac{K\|v\|}{\left(1+\|v\|^{2}\right)^{\frac{\beta}{2}}}\right)^{q}\left(1+\|v\|^{2}\right)^{\frac{\beta(q-1)}{2}} \mathrm{~d} v .
$$

We write $r=\|v\|$, and $\mathrm{d} v=r^{d-1} \mathrm{~d} r \mathrm{~d} \sigma$, where $\mathrm{d} \sigma$ is the measure of the unit sphere $S^{d-1}$ in $\mathbb{R}^{d}$. Then we get

$$
\begin{aligned}
\int_{\mathbb{R}^{d}}|\chi|^{q} \omega^{q-1} \mathrm{~d} v & \leq \int_{S^{d-1}} \int_{0}^{\infty}\left(\frac{r^{2}+3}{3 \beta-2(d+2)} \frac{K r}{\left(1+r^{2}\right)^{\frac{\beta}{2}}}\right)^{q}\left(1+r^{2}\right)^{\frac{\beta(q-1)}{2}} r^{d-1} \mathrm{~d} r \mathrm{~d} \sigma \\
& \leq C \int_{0}^{\infty} \frac{r^{d-1+q}\left(3+r^{2}\right)^{q}}{\left(1+r^{2}\right)^{\frac{\beta}{2}}} \mathrm{~d} r
\end{aligned}
$$

then for $q<\frac{\beta-d}{3}$ we have $\chi \in H_{q}$.

2. In the same way, for $r=\|v\|$ we have

$$
\begin{aligned}
\int_{\mathbb{R}^{d}}|v \chi|^{q} \omega^{q-1} \mathrm{~d} v & \leq \int_{\mathbb{R}^{d}}\left(\frac{\|v\|^{2}+3}{3 \beta-2(d+2)} \frac{K\|v\|^{2}}{\left(1+\|v\|^{2}\right)^{\frac{\beta}{2}}}\right)^{q}\left(1+\|v\|^{2}\right)^{\frac{\beta(q-1)}{2}} \mathrm{~d} v \\
& \leq C \int_{0}^{\infty} \frac{\left(3+r^{2}\right)^{q} r^{2 q+d-1}}{\left(1+r^{2}\right)^{\frac{\beta}{2}}} \mathrm{~d} r
\end{aligned}
$$

then for $q<\frac{\beta-d}{4}$, we get that $v \cdot \chi \in H_{q}$.

\subsection{Study of diffusion coefficient D}

As a consequence of Lemma 5.1 we can define the tensor $D$ by

$$
D=\int_{\mathbb{R}^{d}}(v \otimes v) \frac{\|v\|^{2}+3}{3 \beta-2(d+2)} \frac{K}{\omega} \mathrm{d} v
$$


Proposition 5.3. The tensor $D$ is symmetric and positive definite.

Proof. Let $X \in \mathbb{R}^{d}$ and $Y \in \mathbb{R}^{d}$. Let us denote by $\langle\cdot, \cdot\rangle$ the scalar product in $\mathbb{R}^{d}$ whereas $\langle\cdot, \cdot\rangle_{H}$ is the scalar product associated to the Hilbert space $H$. We have $\langle D X, Y\rangle=\sum_{i, j} D_{i j} X_{i} Y_{j}$ with

$$
D_{i j}=\int_{\mathbb{R}^{d}} \frac{K v_{i}}{\omega} \chi_{j} \omega \frac{\mathrm{d} v}{K}=-\int_{\mathbb{R}^{d}} Q\left(\chi_{i}\right) \chi_{j} \omega \frac{\mathrm{d} v}{K}=-\frac{1}{K}\left\langle Q\left(\chi_{i}\right), \chi_{j}\right\rangle_{H}
$$

Since $Q$ is selfadjoint in $H$, we get $D_{i j}=D_{j i}$. In addition,

$$
\begin{aligned}
\langle D X, X\rangle & =\sum_{i, j} D_{i j} X_{i} X_{j}=-\frac{1}{K} \sum_{i, j}\left\langle Q\left(\chi_{i}\right), \chi_{j}\right\rangle_{H} X_{i} X_{j} \\
& =-\frac{1}{K}\left\langle Q\left(f_{X}\right), f_{X}\right\rangle_{H}=\frac{1}{K} \int_{\mathbb{R}^{d}} \frac{\left|\nabla\left(f_{X} \omega\right)\right|^{2}}{\omega} \mathrm{d} v \\
& \geq 0
\end{aligned}
$$

where $f_{X}(v)=\sum_{i=1}^{d} X_{i} \chi_{i}(v)$.

If $\langle D X, X\rangle=0$ we obtain that $f_{X}(v)=\sum_{i=1}^{d} X_{i} \chi_{i}(v)=\frac{\rho(t, x)}{\omega}$, since $Q$ is linear we have $\sum_{i=1}^{d} X_{i} Q\left(\chi_{i}\right)(v)=$ 0 which leads to $\sum_{i=1}^{d} X_{i} \frac{v_{i}}{\omega}=0$. Since $\left\{\frac{v_{i}}{\omega}\right\}_{1 \leq i \leq d}$ is linearly independant, then for all $i ; 1 \leq i \leq d$ we have $X_{i}=0$ and finally $X=0$.

Lemma 5.4. For $\beta>d+4$, the diffusion tensor $D$ is finite.

Proof. Using (5.3) we can see that

$$
|D| \leq \int_{\mathbb{R}^{d}}\|v\|^{2} \frac{\|v\|^{2}+3}{3 \beta-2(d+2)} \frac{K}{\left(1+\|v\|^{2}\right)^{\frac{\beta}{2}}} \mathrm{~d} v,
$$

therefore, for $\beta>d+4, D$ is finite.

\section{Rigorous asymptotics}

\subsection{Compactness}

Our aim in this section is to study the asymptotic behaviour as $\varepsilon$ goes to zero of the solution $f^{\varepsilon}$ of the rescaled equation (1.4), by proving that $f^{\varepsilon}-\frac{K \rho^{\varepsilon}}{\omega}$ converges weakly to zero. We start with a priori estimate for $f^{\varepsilon}$.

Lemma 6.1. For initial datum $f_{0} \in Y_{\omega}^{p}\left(\mathbb{R}^{2 d}\right)$ where $p \geq 2$ and a positive time $T$.

1. The solution $f^{\varepsilon}$ of $(1.4)$ is bounded in $L^{\infty}\left([0, T] ; Y_{\omega}^{p}\left(\mathbb{R}^{2 d}\right)\right)$ uniformly with respect to $\varepsilon$ since it satisfies

$$
\left\|f^{\varepsilon}(T)\right\|_{Y_{\omega}^{p}}^{p}+\frac{p(p-1)}{\theta(\varepsilon)} \int_{0}^{T} \int_{\mathbb{R}^{2 d}} \frac{\left|\nabla_{v}\left(f^{\varepsilon} \omega\right)\right|^{2}}{\omega}\left(f^{\varepsilon}\right)^{p-2} \omega^{p-2} \mathrm{~d} v \mathrm{~d} x \mathrm{~d} t \leq\left\|f_{0}\right\|_{Y_{\omega}^{p}}^{p} .
$$

2. The density $\rho^{\varepsilon}(t, x)=\int_{\mathbb{R}^{d}} f^{\varepsilon} \mathrm{d} v$ is such that

$$
\left\|\rho^{\varepsilon}(t)\right\|_{p}^{p} \leq \frac{1}{K^{p-1}}\left\|f_{0}\right\|_{Y_{\omega}^{p}}^{p} \quad \text { for all } \quad t \in[0, T] .
$$

3. Then, up to a subsequence, the density $\rho^{\varepsilon}$ converges weakly star in $L^{\infty}\left([0, T] ; L^{p}\left(\mathbb{R}^{d}\right)\right)$ to $\rho$.

4. Up to a subsequence, the sequence $f^{\varepsilon}$ converges weakly star in $L^{\infty}\left([0, T] ; Y_{\omega}^{p}\left(\mathbb{R}^{2 d}\right)\right)$ to $f=\rho(t, x) \frac{K}{\omega}$. 
Proof.

1. Multiplying (1.4) by $\left(f^{\varepsilon}\right)^{p-1} \omega^{p-1}$ and integrating it with respect to $x$ and $v$, we obtain

$$
\int_{\mathbb{R}^{2 d}} \partial_{t} f^{\varepsilon}\left(f^{\varepsilon}\right)^{p-1} \omega^{p-1} \mathrm{~d} v \mathrm{~d} x=\frac{1}{\theta(\varepsilon)} \int_{\mathbb{R}^{2 d}} \nabla_{v} \cdot\left(\frac{1}{\omega} \nabla_{v}\left(f^{\varepsilon} \omega\right)\right)\left(f^{\varepsilon}\right)^{p-1} \omega^{p-1} \mathrm{~d} v \mathrm{~d} x .
$$

An integration by parts in $v$ gives:

$$
\begin{aligned}
\frac{1}{p} \frac{\mathrm{d}}{\mathrm{d} t}\left\|f^{\varepsilon}\right\|_{Y_{\omega}^{p}}^{p} & =-\frac{1}{\theta(\varepsilon)} \int_{\mathbb{R}^{2 d}} \frac{\nabla_{v}\left(f^{\varepsilon} \omega\right) \cdot \nabla_{v}\left(\left(f^{\varepsilon}\right)^{p-1} \omega^{p-1}\right)}{\omega} \mathrm{dvd} x \\
& =-\frac{1}{\theta(\varepsilon)} \int_{\mathbb{R}^{2 d}} \frac{\nabla_{v}\left(f^{\varepsilon} \omega\right) \cdot \nabla_{v}\left(f^{\varepsilon} \omega\right)}{\omega}(p-1)\left(f^{\varepsilon}\right)^{p-2} \omega^{p-2} \mathrm{~d} v \mathrm{~d} x \\
& =-\frac{1}{\theta(\varepsilon)} \int_{\mathbb{R}^{2 d}} \frac{\left|\nabla_{v}\left(f^{\varepsilon} \omega\right)\right|^{2}}{\omega}(p-1)\left(f^{\varepsilon}\right)^{p-2} \omega^{p-2} \mathrm{~d} v \mathrm{~d} x .
\end{aligned}
$$

An integration in time gives (6.1).

2. The Cauchy-Schwarz inequality implies:

$$
\rho^{\varepsilon}(t, x)=\int_{\mathbb{R}^{d}} \frac{f^{\varepsilon} K^{\frac{p-1}{p}} \omega^{\frac{p-1}{p}}}{K^{\frac{p-1}{p}} \omega^{\frac{p-1}{p}}} \mathrm{~d} v \leq\left(\int_{\mathbb{R}^{d}} \frac{\left(f^{\varepsilon}\right)^{p} \omega^{p-1}}{K^{p-1}} \mathrm{~d} v\right)^{\frac{1}{p}}\left(\int_{\mathbb{R}^{d}} \frac{K}{\omega} \mathrm{d} v\right)^{\frac{p-1}{p}},
$$

then,

$$
\int_{\mathbb{R}^{d}}\left(\rho^{\varepsilon}\right)^{p} \mathrm{~d} x \leq \int_{\mathbb{R}^{d}}\left(\int_{\mathbb{R}^{d}} \frac{\left(f^{\varepsilon}\right)^{p} \omega^{p-1}}{K^{p-1}} \mathrm{~d} v\right) \mathrm{d} x
$$

and

$$
\sup _{t>0} \int\left(\rho^{\varepsilon}\right)^{p} \mathrm{~d} x \leq \frac{1}{K^{p-1}} \sup _{t} \iint\left(f^{\varepsilon}\right)^{p} \omega^{p-1} \mathrm{~d} v \mathrm{~d} x
$$

Using (6.1) we obtain

$$
\left\|\rho^{\varepsilon}(t)\right\|_{p}^{p} \leq \frac{1}{K^{p-1}}\left\|f_{0}\right\|_{Y_{\omega}^{p}}^{p}, \text { for all } t \in[0, T] .
$$

3. The previous inequality gives that $\rho^{\varepsilon}$ is bounded in $L^{\infty}\left([0, T], L^{p}\left(\mathbb{R}^{d}\right)\right)$, and Banach-Alaoglu theorem gives that there exist $\rho \in L^{\infty}\left([0, T], L^{p}\left(\mathbb{R}^{d}\right)\right)$ and a subsequence, still denoted by $\rho^{\varepsilon}$ which converges weakly star in $L^{\infty}\left([0, T] ; L^{p}\left(\mathbb{R}^{d}\right)\right)$ to $\rho$.

4. Using (6.1) we have $\left(f^{\varepsilon}\right)$ is a bounded sequence in $L^{\infty}\left([0, T] ; Y_{\omega}^{p}\left(\mathbb{R}^{2 d}\right)\right)$ uniformly with respect to $\varepsilon$, since it satisfies

$$
\left\|f^{\varepsilon}(T)\right\|_{Y_{\omega}^{p}} \leq\left\|f_{0}\right\|_{Y_{\omega}^{p}}
$$

Therefore, there exists $f \in L^{\infty}\left([0, T], Y_{\omega}^{p}\left(\mathbb{R}^{2 d}\right)\right)$ and a subsequence, still denoted by $f^{\varepsilon}$ satisfying

$$
f^{\varepsilon} \stackrel{\varepsilon \rightarrow 0}{\longrightarrow} f \text { in } L^{\infty}\left([0, T], Y_{\omega}^{p}\left(\mathbb{R}^{2 d}\right)\right) \text { weak star. }
$$

Furthermore, multiplying (1.4) by a test funcion $\phi(t, x, v)$ and integrating it with respect to $v$ we obtain

$$
\begin{aligned}
\int_{\mathbb{R}} \int_{\mathbb{R}^{d}} \int_{\mathbb{R}^{d}} Q\left(f^{\varepsilon}\right) \phi \mathrm{d} v \mathrm{~d} x \mathrm{~d} t= & -\theta(\varepsilon) \int_{\mathbb{R}^{d}} \int_{\mathbb{R}^{d}} f_{0} \phi \mathrm{d} v \mathrm{~d} x-\theta(\varepsilon) \int_{\mathbb{R}} \int_{\mathbb{R}^{d}} \int_{\mathbb{R}^{d}} f^{\varepsilon} \partial_{t} \phi \mathrm{d} v \mathrm{~d} x \mathrm{~d} t \\
& -\varepsilon \int_{\mathbb{R}} \int_{\mathbb{R}^{d}} \int_{\mathbb{R}^{d}} v \cdot \nabla_{x} \phi f^{\varepsilon} \mathrm{d} v \mathrm{~d} x \mathrm{~d} t
\end{aligned}
$$

using (6.4) we obtain that

$$
\int_{\mathbb{R}} \int_{\mathbb{R}^{d}} \int_{\mathbb{R}^{d}} Q\left(f^{\varepsilon}\right) \phi \mathrm{d} v \mathrm{~d} x \mathrm{~d} t \longrightarrow 0, \text { as } \varepsilon \rightarrow 0, \text { for all } \phi .
$$


Therefore, we deduce that $f \in \operatorname{Ker}(Q)$ and there exist $C(t, x)$ such that $f=\frac{K C(t, x)}{\omega}$. Now, let us prove that $C(t, x)=\rho(t, x)$.

For $p^{\prime}$ satisfying $\frac{1}{p}+\frac{1}{p^{\prime}}=1$ and for all $\varphi \in C_{c}^{\infty}\left([0, T], Y_{\omega}^{p^{\prime}}\left(\mathbb{R}^{2 d}\right)\right)$, by $(6.4)$ we have

$$
\int_{\mathbb{R}} \int_{\mathbb{R}^{2 d}} f^{\varepsilon} \varphi \omega \mathrm{d} v \mathrm{~d} x \mathrm{~d} t \rightarrow \int_{\mathbb{R}} \int_{\mathbb{R}^{2 d}} \frac{K C(t, x)}{\omega} \varphi \omega \mathrm{d} v \mathrm{~d} x \mathrm{~d} t
$$

Choosing

$$
\varphi(t, x, v)=\frac{\theta(t, x)}{\omega},
$$

where $\theta(t, x) \in C_{c}^{\infty}\left([0, T], L^{p^{\prime}}\left(\mathbb{R}^{d}\right)\right)$, we obtain that $\frac{\theta(t, x)}{\omega} \in Y_{\omega}^{p^{\prime}}\left(\mathbb{R}^{2 d}\right)$.

Substituting (6.7) into (6.6) and using the convergence of $\rho^{\varepsilon}$ we obtain

$$
\int_{\mathbb{R}} \int_{\mathbb{R}^{2 d}} f^{\varepsilon} \varphi \omega \mathrm{d} v \mathrm{~d} x \mathrm{~d} t=\int_{\mathbb{R}} \int_{\mathbb{R}^{d}} \rho^{\varepsilon} \theta \mathrm{d} x \mathrm{~d} t \longrightarrow \int_{\mathbb{R}} \int_{\mathbb{R}^{d}} \rho \theta \mathrm{d} x \mathrm{~d} t, \text { as } \varepsilon \rightarrow 0 .
$$

On another hand, using the convergence of $f^{\varepsilon}$ we have

$$
\int_{\mathbb{R}} \int_{\mathbb{R}^{2 d}} f^{\varepsilon} \varphi \omega \mathrm{d} v \mathrm{~d} x \mathrm{~d} t \longrightarrow \int_{\mathbb{R}} \int_{\mathbb{R}^{d}} \frac{K C(t, x)}{\omega} \theta \mathrm{d} x \mathrm{~d} t, \text { as } \varepsilon \rightarrow 0 .
$$

Since $\int_{\mathbb{R}^{d}} \frac{K}{\omega} \mathrm{d} v=1$, we have

$$
\rho(t, x)=\int_{\mathbb{R}^{d}} \frac{K C(t, x)}{\omega} \mathrm{d} v=C(t, x) .
$$

Which ends the proof of the Lemma.

\subsection{Study of the current}

We recall the definition of the macroscopic current

$$
j^{\varepsilon}(t, x)=\int_{\mathbb{R}^{d}} \frac{v f^{\varepsilon}}{\varepsilon} \mathrm{d} v .
$$

We prove that $j^{\varepsilon}$ has a weak limit as $\varepsilon$ goes to zero.

Lemma 6.2. For $\beta>d+2$ and fixed $\varepsilon>0$ the current $j^{\varepsilon}$ is defined.

Proof. The Cauchy-Schwarz inequality implies:

$$
\left|\int_{\mathbb{R}^{d}} v f^{\varepsilon} \mathrm{d} v\right| \leq \int_{\mathbb{R}^{d}} \frac{\left|v f^{\varepsilon}\right| \sqrt{\omega}}{\sqrt{\omega}} \mathrm{d} v \leq\left(\int_{\mathbb{R}^{d}} \frac{\|\left. v\right|^{2}}{\omega} \mathrm{d} v\right)^{\frac{1}{2}}\left(\int_{\mathbb{R}^{d}}\left|f^{\varepsilon}\right|^{2} \omega \mathrm{d} v\right)^{\frac{1}{2}} .
$$

We deduce that for $\beta>d+2$ the current $j^{\varepsilon}$ is defined.

Lemma 6.3. For $\beta>d+4$, the current $j^{\varepsilon}$ is bounded uniformly with respect to $\varepsilon$ in $L_{\text {loc }}^{2}\left([0, T] \times \mathbb{R}^{d}\right)$.

Proof. We remark that $\frac{v}{\omega}=\frac{1}{-\beta} \nabla_{v}\left(\frac{1}{\left(1+\|v\|^{2}\right)^{\frac{\beta}{2}-1}}\right)$, then

$$
\begin{aligned}
j^{\varepsilon}(t, x)=\frac{1}{\varepsilon} \int_{\mathbb{R}^{d}} \frac{v}{\omega}\left(f^{\varepsilon} \omega\right) \mathrm{d} v & =\frac{1}{-\beta \varepsilon} \int_{\mathbb{R}^{d}} \nabla_{v}\left(\frac{1}{\left(1+\|v\|^{2}\right)^{\frac{\beta}{2}-1}}\right)\left(f^{\varepsilon} \omega\right) \mathrm{d} v \\
& =\frac{1}{\beta \varepsilon} \int_{\mathbb{R}^{d}}\left(1+\|v\|^{2}\right)^{\frac{-\beta}{2}+1} \sqrt{\omega} \frac{\nabla_{v}\left(f^{\varepsilon} \omega\right)}{\sqrt{\omega}} \mathrm{d} v .
\end{aligned}
$$


Cauchy-Schwarz inequality implies,

$$
\left|j^{\varepsilon}\right| \leq c\left(\int_{\mathbb{R}^{d}}\left(1+\|v\|^{2}\right)^{-\beta+2}\left(1+\|v\|^{2}\right)^{\frac{\beta}{2}} \mathrm{~d} v\right)^{\frac{1}{2}}\left(\int_{\mathbb{R}^{d}} \frac{\left|\nabla_{v}\left(f^{\varepsilon} \omega\right)\right|^{2}}{\varepsilon^{2} \omega} \mathrm{d} v\right)^{\frac{1}{2}} .
$$

Using (6.3) with $p=2$, there exists a constant $C$ such that

$$
\int_{0}^{T} \int_{\mathbb{R}^{2 d}} \frac{\left|\nabla_{v}\left(f^{\varepsilon} \omega\right)\right|^{2}}{\varepsilon^{2} \omega} \mathrm{d} v \mathrm{~d} x \mathrm{~d} t \leq C
$$

then for any bounded domain $\Omega \subset \mathbb{R}^{d}$, we have:

$$
\begin{aligned}
\int_{0}^{T} \int_{\Omega}\left|j^{\varepsilon}\right|^{2} \mathrm{~d} x \mathrm{~d} t \leq & \int_{0}^{T} \int_{\Omega} \int_{\mathbb{R}^{d}}\left(1+|| v \|^{2}\right)^{-\beta+2}\left(1+\|v\|^{2}\right)^{\frac{\beta}{2}} \mathrm{~d} v \mathrm{~d} x \mathrm{~d} t \\
& \times \int_{0}^{T} \int_{\Omega} \int_{\mathbb{R}^{d}} \frac{\left|\nabla_{v}\left(f^{\varepsilon} \omega\right)\right|^{2}}{\varepsilon^{2} \omega} \mathrm{d} v \mathrm{~d} x \mathrm{~d} t .
\end{aligned}
$$

Finally, for $\beta>d+4$,

$$
\int_{\mathbb{R}^{d}}\left(1+\|v\|^{2}\right)^{-\beta+2}\left(1+\|v\|^{2}\right)^{\frac{\beta}{2}} \mathrm{~d} v<\infty,
$$

and $j^{\varepsilon}$ is bounded in $L_{l o c}^{2}\left([0, T] \times \mathbb{R}^{d}\right)$, and thus $j^{\varepsilon}$ converges weakly in $L_{l o c}^{2}\left([0, T] \times \mathbb{R}^{d}\right)$.

It remains to identify the limiting flux $j$.

\subsection{Moment method}

Proposition 6.4. Under the assumptions of Theorem 2.2, we identify the limit $\rho(t, x)$ solution to the diffusion equation (2.3) and we conclude that the whole sequence $f^{\varepsilon}$ converges weak star in $L^{\infty}\left([0, T] ; Y_{\omega}^{p}\left(\mathbb{R}^{2 d}\right)\right.$.

Proof. Take any subsequence still denoted by $f^{\varepsilon}$. Let us rewrite a weak formulation of (1.4)

$$
\frac{\mathrm{d}}{\mathrm{d} t} \int_{\mathbb{R}^{2 d}} f^{\varepsilon} \varphi \omega \mathrm{d} v \mathrm{~d} x=\int_{\mathbb{R}^{2 d}} f^{\varepsilon}\left(\partial_{t} \varphi+\frac{1}{\varepsilon} v \cdot \nabla_{x} \varphi+\frac{1}{\varepsilon^{2}} Q(\varphi)\right) \omega \mathrm{d} v \mathrm{~d} x
$$

for all test function $\varphi(t, x, v)$ in the space $C_{c}^{\infty}\left(\mathbb{R} \times \mathbb{R}^{d}, H_{q}\right)$ with $q<\frac{\beta-d}{3}$. In the weak formulation (6.10), to obtain an equation on the density $\rho$, we should take a test function with the following shape $\varphi(t, x, v)=\frac{K \phi(t, x)}{\omega}$, where $\phi$ does not depend on $v$. In equation (6.10) the more singular term vanishes but it still remains a singularity via the advection term. To handle it, we introduce a correction by writing $\varphi(t, x, v)=\frac{K \phi(t, x)}{\omega}+\varepsilon \psi(t, x, v)$, and for the singularities to balance, we take $\psi(t, x, v)$ solution to the auxiliary equation:

$$
Q(\psi)=-\frac{K v \cdot \nabla_{x} \phi(t, x)}{\omega}
$$

which means that $\psi(t, x, v)=\chi \cdot \nabla_{x} \phi(t, x)$, where $\chi=Q^{-1}\left(\frac{-v K}{\omega}\right)$, and $\phi$ is a smooth compactly supported vector test function of $(t, x)$.

Using the expression of $j^{\varepsilon}$ in (6.9), and since by Lemma 6.2, we can write

$$
K \int_{\mathbb{R}^{d}} \int_{\mathbb{R}^{d}} \frac{1}{\varepsilon} f^{\varepsilon} v \cdot \nabla_{x} \phi \mathrm{d} v \mathrm{~d} x=\int_{\mathbb{R}^{d}} j^{\varepsilon} \cdot \nabla_{x} \phi \mathrm{d} x=-\frac{1}{\varepsilon} \int_{\mathbb{R}^{d}} \int_{\mathbb{R}^{d}} f^{\varepsilon} Q(\psi) \omega \mathrm{d} v \mathrm{~d} x,
$$


then, (6.10) becomes

$$
\begin{aligned}
\frac{\mathrm{d}}{\mathrm{d} t} \int_{\mathbb{R}^{d}} \int_{\mathbb{R}^{d}} f^{\varepsilon}\left(\frac{K \phi}{\omega}+\varepsilon \psi\right) \omega \mathrm{d} v \mathrm{~d} x= & \int_{\mathbb{R}^{d}} \int_{\mathbb{R}^{d}} f^{\varepsilon} K \partial_{t} \phi \mathrm{d} v \mathrm{~d} x+\int_{\mathbb{R}^{d}} \int_{\mathbb{R}^{d}} f^{\varepsilon} v \cdot \nabla_{x} \psi \omega \mathrm{d} v \mathrm{~d} x \\
& +\varepsilon \int_{\mathbb{R}^{d}} \int_{\mathbb{R}^{d}} f^{\varepsilon} \partial_{t} \psi \omega \mathrm{d} v \mathrm{~d} x .
\end{aligned}
$$

Integrating the above equation with respect to time, we obtain

$$
\begin{aligned}
-\int_{\mathbb{R}^{d}} \int_{\mathbb{R}^{d}} f^{\varepsilon}(0)\left(\frac{K \phi(0)}{\omega}+\varepsilon \psi(0)\right) \omega \mathrm{d} v \mathrm{~d} x= & \varepsilon \int_{0}^{\infty} \int_{\mathbb{R}^{d}} \int_{\mathbb{R}^{d}} f^{\varepsilon} \partial_{t} \psi \omega \mathrm{d} v \mathrm{~d} x \mathrm{~d} t \\
& +\int_{0}^{\infty} \int_{\mathbb{R}^{d}} \int_{\mathbb{R}^{d}} f^{\varepsilon} K \partial_{t} \phi \mathrm{d} v \mathrm{~d} x \mathrm{~d} t \\
& +\int_{0}^{\infty} \int_{\mathbb{R}^{d}} \int_{\mathbb{R}^{d}} f^{\varepsilon} v \cdot \nabla_{x} \psi \omega \mathrm{d} v \mathrm{~d} x \mathrm{~d} t .
\end{aligned}
$$

The trace at $t=0$ has a meaning, thanks to a trace formula for function in $Y$ which is proven in Lemma 3.3 .

Which reads

$$
\begin{aligned}
-K \int_{\mathbb{R}^{d}} \int_{\mathbb{R}^{d}} f^{\varepsilon}(0) \phi(0) \mathrm{d} v \mathrm{~d} x & -K \int_{0}^{\infty} \int_{\mathbb{R}^{d}} \int_{\mathbb{R}^{d}} f^{\varepsilon} \partial_{t} \phi \mathrm{d} v \mathrm{~d} x \mathrm{~d} t \\
= & \varepsilon \int_{\mathbb{R}^{d}} \int_{\mathbb{R}^{d}} f^{\varepsilon}(0) \psi(0) \omega \mathrm{d} v \mathrm{~d} x+\varepsilon \int_{0}^{\infty} \int_{\mathbb{R}^{d}} \int_{\mathbb{R}^{d}} f^{\varepsilon} \partial_{t} \psi \omega \mathrm{d} v \mathrm{~d} x \mathrm{~d} t \\
& +\int_{0}^{\infty} \int_{\mathbb{R}^{d}} \int_{\mathbb{R}^{d}} f^{\varepsilon} v \cdot \nabla_{x} \psi \omega \mathrm{d} v \mathrm{~d} x \mathrm{~d} t .
\end{aligned}
$$

Using the weak convergence of $\rho^{\varepsilon}$, we deduce that the left hand side in (6.13) converges, more precisely

$$
K \int_{\mathbb{R}^{d}} \int_{\mathbb{R}^{d}} f^{\varepsilon}(0) \phi(0) \mathrm{d} v \mathrm{~d} x=K \int_{\mathbb{R}^{d}} \rho^{\varepsilon}(0) \phi(0) \mathrm{d} x \stackrel{\varepsilon \rightarrow 0}{\longrightarrow} K \int_{\mathbb{R}^{d}} \rho(0) \phi(0) \mathrm{d} x
$$

and

$$
K \int_{0}^{\infty} \int_{\mathbb{R}^{d}} \int_{\mathbb{R}^{d}} f^{\varepsilon} \partial_{t} \phi \mathrm{d} v \mathrm{~d} x \mathrm{~d} t=K \int_{0}^{\infty} \int_{\mathbb{R}^{d}} \rho^{\varepsilon} \partial_{t} \phi \mathrm{d} x \mathrm{~d} t \stackrel{\varepsilon \rightarrow 0}{\longrightarrow} K \int_{0}^{\infty} \int_{\mathbb{R}^{d}} \rho \partial_{t} \phi \mathrm{d} x \mathrm{~d} t .
$$

It remains to pass to the limit $\varepsilon \rightarrow 0$ in the right hand side in (6.13). We show that the first line in the right hand side in (6.13) goes to zero when $\varepsilon \rightarrow 0$. Indeed, by Hölder inequality, we have for $p>1, q>1$ satisfying $\frac{1}{p}+\frac{1}{q}=1$

$$
\begin{aligned}
\left|\varepsilon \int_{\mathbb{R}^{d}} \int_{\mathbb{R}^{d}} f^{\varepsilon}(0) \psi(0) \omega \mathrm{d} v \mathrm{~d} x\right| & \leq \varepsilon \int_{\mathbb{R}^{d}} \int_{\mathbb{R}^{d}}\left|f^{\varepsilon}(0) \chi \cdot \nabla_{x} \phi(0) \omega\right| \mathrm{d} v \mathrm{~d} x \\
& \leq \varepsilon\left\|f_{0} \omega^{\frac{p-1}{p}}\right\|_{p}\left\|\chi \cdot \nabla_{x} \phi(0) \omega^{\frac{q-1}{q}}\right\|_{q} \\
& \leq \varepsilon\left\|f_{0}\right\|_{Y_{\omega}^{p}}\left\|\chi \cdot \nabla_{x} \phi(0)\right\|_{Y_{\omega}^{q}}
\end{aligned}
$$

Since $f_{0} \in Y_{\omega}^{p}\left(\mathbb{R}^{2 d}\right)$, and since by Lemma 5.2, for $1<q<\frac{\beta-d}{4}, \chi \in Y_{\omega}^{q}\left(\mathbb{R}^{2 d}\right)$, we deduce from (6.14) that

$$
\varepsilon \int_{\mathbb{R}^{d}} \int_{\mathbb{R}^{d}} f^{\varepsilon}(0) \psi(0) \omega \mathrm{d} v \mathrm{~d} x \longrightarrow 0, \text { when } \varepsilon \rightarrow 0
$$


Similarly for $1<q<\frac{\beta-d}{4}$ and by Hölder inequality, we obtain for $(p, q)$ satisfying $\frac{1}{p}+\frac{1}{q}=1$,

$$
\begin{aligned}
\left|\varepsilon \int_{0}^{\infty} \int_{\mathbb{R}^{d}} \int_{\mathbb{R}^{d}} f^{\varepsilon} \partial_{t} \psi \omega \mathrm{d} v \mathrm{~d} x \mathrm{~d} t\right| & \leq \varepsilon \int_{0}^{\infty} \int_{\mathbb{R}^{d}} \int_{\mathbb{R}^{d}}\left|f^{\varepsilon} \partial_{t} \psi \omega\right| \mathrm{d} v \mathrm{~d} x \mathrm{~d} t \\
& \leq \varepsilon \int_{0}^{\infty}\left\|f^{\varepsilon}\right\|_{Y_{\omega}^{p}}\left\|\chi \cdot \nabla_{x}\left(\partial_{t} \phi\right)\right\|_{Y_{\omega}^{q}} \mathrm{~d} t \\
& \longrightarrow 0 \text { as } \varepsilon \rightarrow 0 .
\end{aligned}
$$

The last integral in (6.13) becomes

$$
\int_{0}^{\infty} \int_{\mathbb{R}^{d}} \int_{\mathbb{R}^{d}} f^{\varepsilon} v \cdot \nabla_{x} \psi \omega \mathrm{d} v \mathrm{~d} x \mathrm{~d} t=\int_{0}^{\infty} \int_{\mathbb{R}^{d}} \int_{\mathbb{R}^{d}} f^{\varepsilon}\left(v \otimes \chi: D^{2} \phi\right) \omega \mathrm{d} v \mathrm{~d} x \mathrm{~d} t,
$$

denoting $A: B=\operatorname{tr}\left(A B^{T}\right)=\sum_{i, j=1}^{d} A_{i j} B_{i j}$ for two $d \times d$ matrices $\mathrm{A}$ and $\mathrm{B}$.

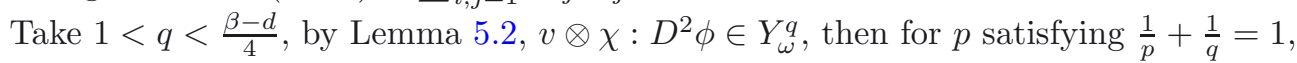

$$
I^{\varepsilon}=\int_{0}^{\infty}\left\langle f^{\varepsilon} ; v \otimes \chi: D^{2} \phi\left\langle_{Y_{\omega}^{p}, Y_{\omega}^{q}} \mathrm{~d} t,\right.\right.
$$

where $\langle\cdot, \cdot\rangle_{Y_{\omega}^{p}, Y_{\omega}^{q}}$ denotes the duality bracket between $Y_{\omega}^{p}$ and $Y_{\omega}^{q}$.

By taking the limit $\varepsilon \rightarrow 0$ we have

$$
I^{\varepsilon} \longrightarrow \int_{0}^{\infty}\left\langle\frac{K \rho}{\omega} ; v \otimes \chi: D^{2} \phi\right\rangle_{Y_{\omega}^{p}, Y_{\omega}^{q}} \mathrm{~d} t
$$

Note that this is possible since $\beta>d+4$ and that this argument degenerates for $\beta=d+4$.

Finally, combining all this limits we obtain

$$
\begin{aligned}
-K \int_{\mathbb{R}^{d}} \rho(0) \phi(0) \mathrm{d} x= & K \int_{0}^{\infty} \int_{\mathbb{R}^{d}} \partial_{t} \phi \rho \mathrm{d} x \mathrm{~d} t \\
& +\int_{0}^{\infty} \int_{\mathbb{R}^{d}} \int_{\mathbb{R}^{d}}\left(v \otimes \chi: D^{2} \phi\right) \rho K \mathrm{~d} v \mathrm{~d} x \mathrm{~d} t,
\end{aligned}
$$

which is nothing but the weak formulation of the drift-diffusion equation satisfied by $\rho$, with initial datum $\rho_{0}$. By uniqueness of the limit, the whole sequence $f^{\varepsilon}$ converges. This concludes the proof.

Acknowledgements. We thank Philippe Laurençot for fruitful discussion.

\section{REFERENCES}

[1] C. Bardos, R. Santos and R. Sentis, Diffusion approximation and computation of the critical size. Numerical Solutions of Nonlinear Problems. INRIA, Rocquencourt (1984).

[2] N. Ben Abdallah, A. Mellet and M. Puel, Anomalous diffusion limit for kinetic equations with degenerate collision frequency. M3AS 21 (2011) 2249-2262.

[3] N. Ben Abdallah, A. Mellet and M. Puel, Fractional diffusion limit for collisional kinetic equations: a Hilbert expansion approach. KRM 4 (2011) 873-900.

[4] A. Bensoussan, J.-L. Lions and G. Papanicolaou, Boundary layers and homogenization of transport processes. Publ. Res. Inst. Math. Sci. 15 (1979) 53-157.

[5] P. Cattiaux, E. Nasreddine and M. Puel, Diffusion limit of Fokker-Planck equation with heavy tails equilibria: a probabilistic approach including anomalous rate (preprint).

[6] L. Cesbron, A. Mellet and K. trivisa, Anomalous transport of particles in plasma physics. Appl. Math. Lett. 25 (2012) 23442348. 
[7] P. Degond, Global existence of smooth solutions for the Vlasov-Fokker-Planck equation in one and two spaces dimensions, vol. 19 of Ann. Sci. E.N.S 4e Ser. (1986) 519-542.

[8] P. Degond. Macroscopic limits of the Boltzmann equation: a review. Modeling and computational methods for kinetic equations, vol. 357 of Model. Simul. Sci. Eng. Technol. Birkhauser Boston, Boston, MA (2004).

[9] P. Degond and P. Mas-Gallic, Existence of solutions and diffusion approximation for a model Fokker-Planck equation. Proc. of the conference on mathematical methods applied to kinetic equations, Paris, 1985. Transp. Theory Stat. Phys. 16 (1987) 589-636.

[10] P. Degond, T. Goudon and F. Poupaud, Diffusion limit for nonhomogeneous and non-micro-reversible processes. Indiana Univ. Math. J. 49 (2000) 1175-1198.

[11] P. garbaczewski, V. Stephanovich and D. Kedzierski, Heavy-tailed targets and (ab)normal asymptotics in diffusive motion. Physica A 390 (2011) 990-1008.

[12] E. Larsen and J. Keller. Asymptotic solution of neutron transport problems for small mean free paths. J. Math. Phys. 15 (1974) $75-81$.

[13] J.L. Lions, Equations différentielles opérationnelles et problèmes aux limites. Vol. 9. Springer, Berlin 1961 (2003) 371-398.

[14] E. Lutz, Anomalous diffusion and Tsallis statistics in an optical lattice. Phys. Rev. A 67 (2003) 051402.

[15] E. Lutz, Power-law tail distributions and nonergodicity. Phys. Rev. Lett. 93 (2004) 190602.

[16] A. Mellet, Fractional diffusion limit for collisional kinetic equations: a moments method. Indiana Univ. Math. J. 59 (2010) $1333-1360$.

[17] A. Mellet, S. Mishler and C. Mouhot, Fractional diffusion limit for collisional kinetic equations. Arch. Ration. Mech. Anal. 199 (2011) 493-525.

[18] P.L. Lions and G. Toscani. Diffusive limits for finite velocity Boltzmann kinetic models. Rev. Mat. Iberoamer. 13 (1997) $473-513$. 